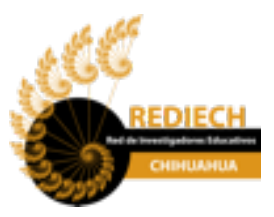

Red de Investigadores Educativos Chihuahua A.C. Chihuahua, México www.rediech.org

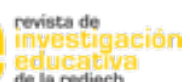

de la rediech

ISSN: 2007-4336

ISSN-e: 2448-8550

http://www.rediech.org/ojs/2017/index.php/ie rie rediech/index

Ricardo López Salazar

Elizabeth Bautista Flores

Óscar Arturo Sánchez Carlos

2019

\title{
INTERESES EDUCATIVOS Y EXPECTATIVAS LABORALES Y PROFESIONALES DE LOS ESTUDIANTES DE NIVEL MEDIO SUPERIOR EN LA REGIÓN NOROESTE DE CHIHUAHUA: UN ANÁLISIS POR GÉNERO
}

IE Revista de Investigación Educativa de la REDIECH, 10(19), pp. 257-274.

DOI: http://dx.doi.org/10.33010/ie_rie_rediech.v10i19.720

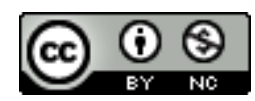

Esta obra está bajo licencia internacional

Creative Commons Reconocimiento-NoComercial 4.0. 


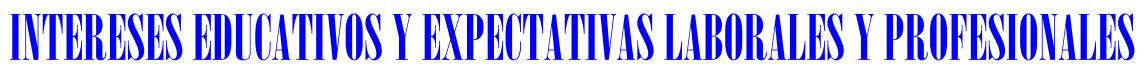

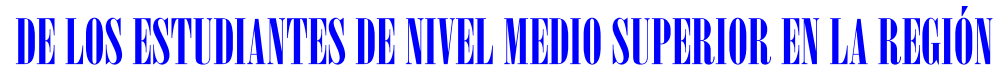

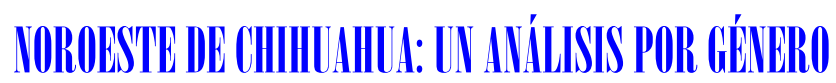

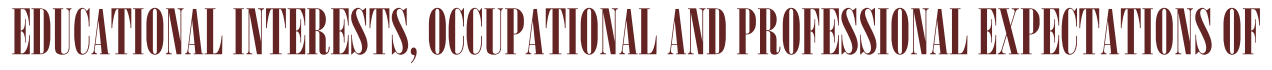

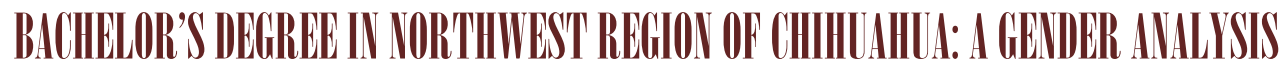

\author{
LÓPEZ SALAZAR Ricardo \\ BAUTISTA FLORES Elizabeth \\ SÁNCHEZ CARLOS Óscar Arturo
}

Recepción: mayo 23 de 2019 | Aprobado para publicación: agosto 27 de 2019

DOI: http://dx.doi.org/10.33010/ie_rie_rediech.v10i19.720

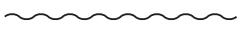

Ricardo López Salazar. Profesor-investigador de tiempo completo titular C en la Universidad Autónoma de Ciudad Juárez, División Multidisciplinaria Nuevo Casas Grandes, Chihuahua, México. Es doctor en Educación con Especialidad en Desarrollo Regional por el Centro de Investigación en Alimentación y Desarrollo. Ha publicado diversos artículos sobre pobreza y políticas públicas en revistas especializadas y tiene participaciones como dictaminador de artículos. Pertenece al Sistema Nacional de Investigadores del Consejo Nacional de Ciencia y Tecnología Nivel 1. Obtuvo el segundo lugar del premio FA0-FLACS0 (2018) "Investigación en innovación de políticas públicas para la seguridad alimentaria y nutricional" en la categoría de artículo publicado. Correo electrónico: ricardo.lopez@uacj.mx. ID: http://orcid.org/0000-0002-0769-5330.

Elizabeth Bautista Flores. Profesora-investigadora de la Universidad Autónoma de Ciudad Juárez, Chihuahua, México. Realizó una estancia de investigación en la Universidad Estatal de Ponta Grossa, Brasil. Realizó estudios de Doctorado en Ciencias Sociales con Especialidad en Comunicación y Política por la Universidad Autónoma Metropolitana Unidad Xochimilco, México; máster en Dirección, Estrategia y Gestión de la Innovación en la Universidad Autónoma de Barcelona y Carlos III de Madrid, España; y Licenciatura y Maestría en Ciencias de la Comunicación en la Facultad de Ciencias Políticas y Sociales de la Universidad Nacional Autónoma de México. Pertenece al Sistema Nacional de Investigadores del Conacyt. Correo electrónico: elizabeth.bautista@uacj.mx. ID: http://orcid.org/0000-00022197-1493.

Óscar Arturo Sánchez Carlos. Profesor de tiempo completo en la Universidad Autónoma de Ciudad Juárez, División Multidisciplinaria Nuevo Casas Grandes, Chihuahua, México. Es doctor en Ciencias por el Colegio de Posgraduados. Participa en los programas de Ingeniería en Agronegocios y Mercadotecnia, donde imparte cursos del área económica, agronómica, control de calidad y matemática. Ha realizado diversos estudios e investigaciones en el noroeste de Chihuahua sobre temas hortícolas y con la comunidad menonita. Actualmente es investigador invitado por el Centro de Investigaciones Económicas, Sociales y Tecnológicas de la Agroindustria y la Agricultura Mundial de la Universidad Autónoma de Chapingo. Correo electrónico: oscar.sanchez@uacj.mx. ID: http://orcid.org/00000002-9154-9810. 


\title{
Resumen
}

En este artículo se exponen y analizan los resultados de una encuesta aplicada entre 2013 y 2014 a estudiantes que cursaron el último año del nivel medio superior en la región conocida como noroeste de Chihuahua (dicha región incluye los municipios de Ascensión, Buenaventura, Janos, Galeana, Casas Grandes, Nuevo Casas Grandes, Zaragoza y Gómez Farías). El ejercicio tuvo como objetivo conocer los intereses educativos y las expectativas laborales y profesionales de acuerdo con el género. Los resultados obtenidos permitieron identificar las tendencias de elección profesional y las diferencias económico-sociales e incluso aspiracionales. Resalta que los jóvenes reciben, en su mayoría, el apoyo de los padres de familia; no obstante, resulta interesante las respuestas vertidas en relación con continuar con los estudios universitarios, ya que mientras los hombres no asignan importancia a la obtención de un título, las mujeres consideran un plan de vida que incluye egresar de licenciatura y continuar sus estudios de posgrado. Sin embargo, las expectativas laborales de las mujeres, medidas a través del ingreso salarial, tienden a ser menores que la de los hombres, ya que esta no se eleva a más de cinco mil pesos por mes. Se concluye que el género influye de manera determinante en la elección del programa de licenciatura, así como en las expectativas laborales y profesionales.

\section{Palabras clave: GÉNERO, INTERESES EDUCATIVOS, EXPECTATIVAS LABORALES Y PROFESIONALES, ESTUDIANTES DE NIVEL MEDIO SUPERIOR, NOROESTE DE CHIHUAHUA.}

\begin{abstract}
In this article is exposed and analyzed the results of a survey applied between 2013 and 2014, to students who attended the last year of the upper middle school level in the region known as Northwest Chihuahua (this region includes the municipalities of Ascension, Buenaventura, Janos, Galeana, Casas Grandes, Nuevo Casas Grandes, Zaragoza and Gomez Farias). The objective of this work was to know the educational interests, the labor and professional expectations according to the gender. The results obtained allowed to identify the tendencies of professional choice, the economic-social differences and even aspirational. It emphasizes that young people receive mostly the support of parents, but it is interesting the answers expressed in relation to continue with the university studies, because while the men do not assign great importance to obtain a grade, women consider a life plan that includes graduating from a bachelor's degree and continue their graduate courses. However, labor expectations of women measured through wage income tend to be lower than men, since it does not rise to more than five thousand pesos per month. It is concluded that gender influences in a decisive way in
\end{abstract}


the choice of the bachelor's degree program, the labor and professional expectations.

Keywords: GENDER, EDUCATIONAL INTERESTS, LABOR AND

PROFESSIONAL EXPECTATIONS, STUDENTS OF THE UPPER MIDDLE SCHOOL LEVEL, NoRTHWESTERN CHIHUAHUA.

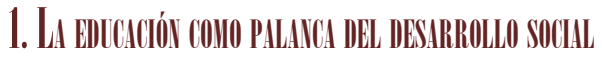

LOS cambios suscitados a nivel mundial a inicios de 1980, que derivó en la instauración de un nuevo orden político y económico a escala planetaria sustentado en la liberalización de mercados y el estímulo a la competencia y la competitividad, también se trasladó hacia el sector educativo, el cual, en esta nueva visión, adquirió un doble propósito. Por un lado, es innegable que una sociedad educada representa una de las premisas más importantes sobre la cual versa la construcción de los estados-nación modernos. De esta manera, se espera que los miembros de la sociedad, al adquirir una educación de calidad, se conviertan en un poderoso instrumento que favorezca la conformación de estados más igualitarios, incluyentes, democráticos y prósperos (Meyer, 1977). Por otro lado, bajo el modelo de economía neoliberal, los países se convierten en agentes activos que compiten por atraer inversiones $y$, por ende, contar con personas altamente cualificadas (educadas sobre todo a nivel formal) representa uno de los elementos diferenciadores para insertarse con éxito en las nuevas dinámicas globales de desarrollo y crecimiento económico (Lewin, 1993).

Es por ello que la educación, durante el siglo pasado y el actual, ha sido catalogada como un bien público (en especial la educación básica); por lo tanto, proveerla es responsabilidad de las autoridades en todas partes del mundo. Así, la educación básica posee las características comunes de los bienes públicos como de irrestricto y libre acceso para toda la población, independientemente de su estrato social, según lo declarado por la Organización de las Naciones Unidas para la Educación, la Ciencia y la Cultura (UNESCO) en 2006.

Con el devenir del siglo anterior, la concepción sobre qué es la educación básica se ha ido transformando, ya que anteriormente se consideraba a la misma hasta el nivel de instrucción primaria; no obstante, en buena parte de los países desarrollados y en vías de desarrollo se incluyó al nivel secundario y la preparatoria. La lógica que subyace a la inclusión de más niveles como parte de la educación básica se orienta hacia el incremento en la cobertura y la matrícula de estudiantes. En otras palabras, al incluir más niveles dentro de la educación básica, disminuyen los costos para los individuos; como un efecto concatenado debe incrementarse la matrícula, posteriormente los egresados y con ello mayores tasas de escolaridad en promedio.

De esta manera, bien se podría decir que el círculo virtuoso de la educación y su impacto en la sociedad se inicia con una preocupación del Estado hacia su provisión, que sin lugar a duda debe incluir la participación y el empoderamiento de la sociedad. A la par, debe gestarse un proceso contrario a la credencialización (incremento de la tasa de graduados, pero con habilidades poco significativas) que redunde en 
personas con capacidades adecuadas al contexto dentro del cual se desenvuelven y que estos generen efectos de diseminación de conocimiento hacia la sociedad misma (López y Bautista, 2015).

Es tan importante la educación en el mundo actual que representa uno de los elementos clave para la conformación del índice de desarrollo humano (IDH), el cual, cabe decir, es uno de los más importantes para la medición del avance y/o estancamiento del bienestar de los países a nivel mundial. En particular, el IDH, creado en 1990 por el paquistaní Mahbubul Haq y el hindú Amartya Sen y que actualmente es utilizado por la Organización de las Naciones Unidas (ONU) para medir el desarrollo anual de los países, utiliza dos indicadores de educación: el primero es la tasa de alfabetización de adultos y el segundo la tasa de matriculación combinada (Haq, 1999).

De acuerdo con datos de la Organización para la Cooperación y el Desarrollo Económicos (OCDE), México registró incrementos significativos en los índices de matriculación de jóvenes; sin embargo, se mantenía en niveles inferiores al promedio de los otros miembros de este organismo mundial.

De 2005 a 2012 la población de 25 a 34 años en México que habían alcanzado la educación media superior aumentó en 8 puntos porcentuales, de $38 \%$ a $46 \%$. No obstante, este porcentaje es mucho menor que el promedio de la OCDE de $83 \%$. Sólo uno de tres adultos de 25 a 64 años de edad terminó ese nivel de educación. México está aumentando el nivel de adolescentes de 15 a 19 años que están en educación: del $48 \%$ al $54 \%$ entre 2005 y 2013. A pesar de este aumento, en 2013 México fue uno de sólo dos países de la OCDE y asociados (el segundo fue Colombia) donde menos de $60 \%$ de los jóvenes de 15 a 19 años estaban inscritos en el sistema educativo [OCDE, 2015, p. 1].

De tal manera que el desarrollo de instrumentos como el IDH, que permite la comparación entre países a nivel mundial, ha puesto en el debate qué tipo de acciones, programas, orientación y objetivos debe tener la educación básica y la educación privada, por lo que, actualmente, el tema educativo representa uno de los más polarizados y que mayor divergencia de opiniones registran en el marco de la agenda pública. Sin embargo, dichas divergencias se centran mayormente en el cómo y no necesariamente en el qué, aspecto que se puede considerar como normal en el marco de una sociedad plural y democrática. Es decir, el objetivo de la educación debe orientarse a dotar de capacidades significativas al individuo para que a través de estas tenga mayores posibilidades de inclusión y desarrollo. Pero, ¿cómo lograr lo anterior? Sin duda ha ocasionado opiniones encontradas y álgidas entre los tomadores de decisiones.

Conforme los individuos logran más educación incrementan su productividad y perciben mejores salarios; cuanto más alto es el nivel educativo de los individuos, mayor es la compensación salarial que consiguen y por tanto los estudios de posgrado son los que muestran las más altas tasas de rendimientos [Villareal, 2018, p. 283].

En particular uno de los puntos más discutidos por las diferentes teorías en el rendi260 miento de la escolaridad se refiere a cómo elevar el nivel educativo, que en un primer 
momento se circunscribió a la inclusión de más niveles dentro de la educación básica. Sin embargo, además de los beneficios naturales de lo anterior, también genera un enorme costo para los estados, aspecto que de manera inmediata se tradujo en un debate perenne sobre qué nivel de gasto público es el idóneo que debe asignarse a la educación. 0 , dicho de otra manera, qué porcentaje del producto interno bruto (PIB) se debe asignar a todo el entramado de instituciones relacionadas con la educación para que esta se traduzca en calidad y no solamente cantidad.

Entre 2005 y 2012, el gasto anual en México por instituciones de educación primaria y secundaria aumentó $19 \%$, en tanto que el número de estudiantes se incrementó $7 \%$. Como resultado, el gasto por estudiante se elevó $11 \%$ (el promedio de la OCDE, que incluye educación postsecundaria no terciaria, fue de $21 \%$ ). Durante este periodo, casi todos los países con un gasto por estudiante por debajo del promedio de la OCDE mostraron aumentos sustanciales en el gasto por estudiante, de $+55 \%$ en Turquía a $+110 \%$ en Brasil [OCDE, 2015, p. 3].

En ese sentido, comúnmente se asocia que el gasto público o gubernamental en el sector educativo es uno de los que mayores rendimientos genera (Villarreal, 2018) $\mathrm{y}$, por ende, los niveles del mismo deben crecer o al menos mantenerse. Con base en lo anterior, si analizamos el promedio del gasto público como porcentaje del PIB, con base en datos proporcionados por el Banco Mundial (2019), en el sector educativo a nivel mundial durante el periodo 1999-2015 encontramos que la tendencia es creciente, con algunos altibajos en 2000 (3.95\%), 2002 y 2004 (4.02\% y 4.03\%, respectivamente). Similar tendencia es posible identificar para Latinoamérica y la Unión Europea, si bien destaca el incremento de 2007-2009 para Latinoamérica, alcanzando un máximo de $6.55 \%$ en 2009, para posteriormente disminuir a niveles del $5 \%$. En el caso de México, comparativamente hablando el nivel de gasto educativo es el más bajo al iniciar el periodo, para incrementarse de manera gradual hasta situarse en el nivel promedio de AL, la UE y ser ligeramente superior al promedio mundial (véase figura 1).

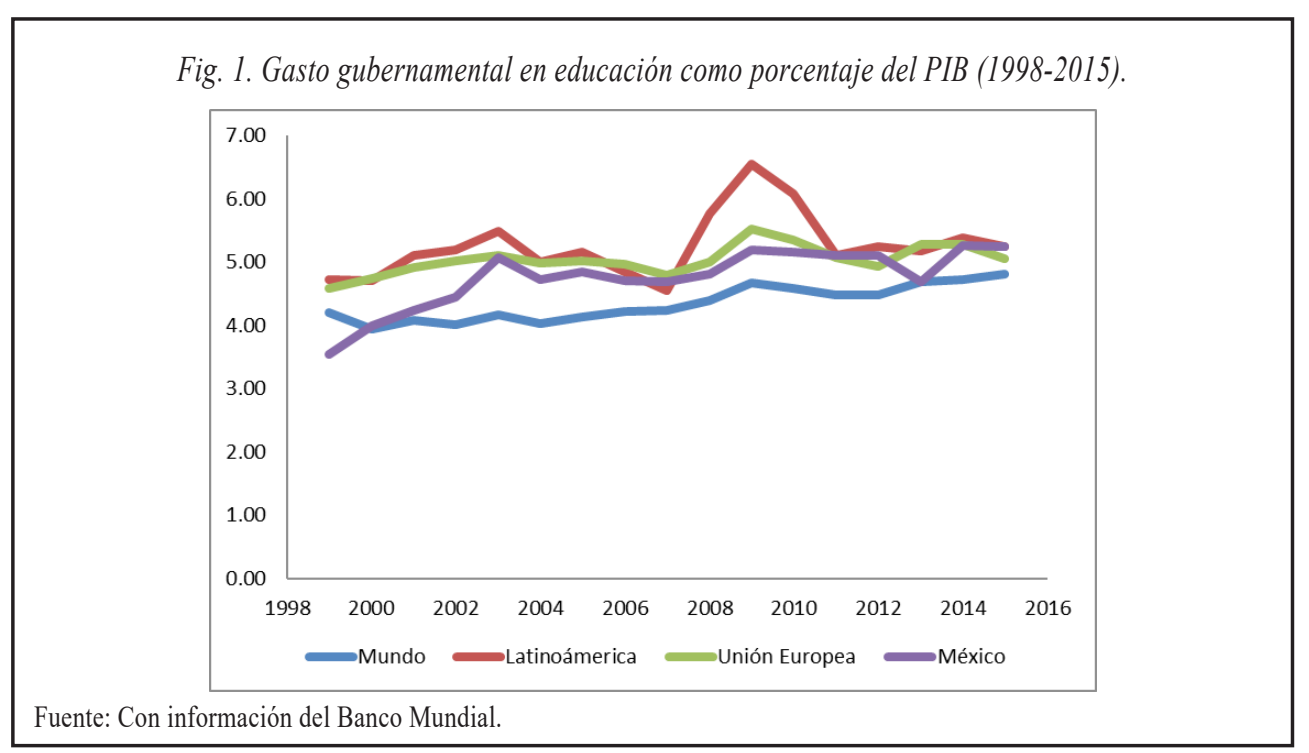


Otro aspecto relevante del tema educativo y su vinculación con la sociedad se encuadra en los tipos de conocimientos que se deben generar dentro de las aulas, los laboratorios y en general en todos los espacios de generación de conocimiento y que este sea útil para la generación de riqueza y bienestar para la sociedad. Como se mencionó al inicio de esta sección, en el mundo actual, caracterizado por la intensa competencia entre regiones, estados y países, el nivel educativo se ha posicionado como un elemento central que significa la diferencia entre qué y cuáles regiones son más atractivas para la recepción de nuevas inversiones, empresas y actividades productivas. Por lo que, actualmente, la educación y la generación de sinergias con los ámbitos públicos y privados (fundamentalmente con el mundo empresarial), en aras de incrementar la competitividad de las regiones, ha sido uno de los aspectos más analizados, sobre todo en el marco de la economía del conocimiento y los sistemas regionales de innovación (Serdyukov, 2017).

Quizá la última gran acción destinada a impulsar a la educación en el mundo es su inclusión dentro de los objetivos de desarrollo sostenible (ODS) de la ONU. De las numerosas metas trazadas en ese punto hacia 2030 destacan la inclusión de un mayor número de niños y niñas a educación de calidad, mejorar la especialización técnica, teórica y práctica de los alumnos, incentivar el desarrollo de mejores docentes e incrementar considerablemente las becas en los países menos desarrollados (UNESCO, 2017).

Sin embargo, a pesar de que seguramente persistirán diferencias en relación a cómo traducir el gasto público, los programas, los modelos y las instituciones en una educación de mayor calidad, debe permanecer la premisa sugerida por Meyer (1977), para quien la educación representa en sí un acto social; es decir, es a través de ella como los individuos nos preparamos para ser parte de una sociedad específica, y que para la conformación de sociedades modernas las instituciones educativas deben poseer un alto grado de desarrollo. Para lograr una sociedad moderna, con todo lo que esto involucra, es necesario contar con instituciones educativas altamente capacitadas para que los individuos que emanen de las mismas sean capaces de formar redes de convivencia que busquen el bien común.

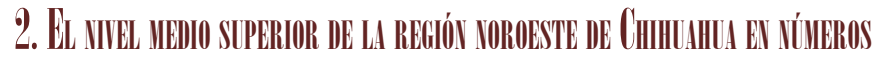

Durante el ciclo escolar 2016-2017, la matrícula registrada a nivel nacional del nivel medio superior (EMS) ascendió a 5.1 millones de alumnos, que representó 14\% del total de estudiantes matriculados. Según el INEE, la EMS es el tipo educativo que ha registrado el mayor crecimiento sostenido en los últimos 15 años. La matrícula pasó de alrededor de 3.1 millones de alumnos a poco más de 5.1 millones; es decir, tuvo una tasa de crecimiento promedio anual de 3.4\% (INEE, 2018, p. 5).

El crecimiento de la EMS se explica en buena parte por su reciente categorización como obligatoria al reformarse los artículos tercero y trigésimo primero en 2012, lo cual incluyó mayores obligaciones por parte del Estado mexicano en cuanto a la provisión de la misma, y se dirigió hacia el cumplimiento de la cobertura universal 262 hasta la educación superior prevista para el ciclo escolar 2021-2022 (INEE, 2018). 
Por su parte, en el estado de Chihuahua se registraron 150,535 alumnos en el citado nivel, que arroja una media de 273 alumnos inscritos por plantel, un poco menor a los 289 alumnos promedio a escala nacional. A nivel regional, en el periodo 20132014 las ciudades de Juárez y Chihuahua albergaron entre ambos a 99,047 alumnos, que equivale a 70\% del total, distribuidos en 141 y 101 planteles, respectivamente, que arroja una media de 388 estudiantes por unidad, ampliamente superior a la media estatal y nacional. La cara opuesta la exhiben veinte municipios que solamente tienen un espacio educativo de nivel medio superior, siendo Huejotitán el de menor alumnado, con 28, según el Sistema Nacional de Información Estadística Educativa (SNIEE, 2016).

La región noroeste del estado, compuesta por los municipios de Ascensión, Buenaventura, Janos, Galeana, Casas Grandes, Nuevo Casas Grandes, Zaragoza y Gómez Farías, contaban con 34 planteles dedicados a atender la demanda educativa de bachillerato. El número total de estudiantes matriculados ascendió a 6,171, destacando el municipio de Nuevo Casas Grandes, que albergó 10 planteles y 2,785 estudiantes, equivalentes a $45 \%$ del total la región de estudio. Sin embargo, lo anterior apenas representó el $4.37 \%$ del total del alumnado de la misma categoría registrada en el estado de Chihuahua (SNIEE, 2013).

La EMS ha mostrado un incremento en el acceso de jóvenes, impulsado por las reformas a la Constitución y su consecuente obligatoriedad. No obstante, en función de las estadísticas estatales y nacionales, el incremento en la matriculación de los jóvenes solo representa un primer estadio hacia la consolidación y elevación del nivel de escolaridad en México. Por ejemplo, incrementar la eficiencia terminal podría ser un primer paso, ya que según datos del INEE (2018) en Chihuahua alcanza $62.3 \%$ y para todo el país en $66 \%$. El fenómeno anterior ayuda a comprender por qué, a pesar del incremento en la cobertura y en la matrícula de la EMS en 20172018, la escolaridad promedio en México se situó en apenas 9.4 años, justamente en secundaria terminada.

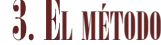

El diseño metodológico de la investigación es el siguiente: primeramente, se elaboró un cuestionario consistente en 34 preguntas, de las cuales 28 fueron cerradas y seis se dejaron abiertas para que el encuestado respondiera lo que mejor reflejara su opinión. Se recopilaron 801 encuestas distribuidas entre hombres y mujeres inscritos en el último año escolar de bachillerato de la región de estudio durante el ciclo escolar 2014-2015. Las escuelas que participaron en este ejercicio se muestran en la tabla 1.

El periodo de aplicación para la primera etapa fue en los meses de agosto y septiembre de 2014) a estudiantes del $5^{\circ}$ semestre del nivel bachillerato. Previamente se acordó con las autoridades fecha y hora. El cuestionario fue autoaplicado y tenía una duración para responder de 10 minutos como mínimo.

Las variables con las cuales se trabajó fueron:

- Origen y nivel de gastos. Se buscó identificar el perfil económico de los estudiantes, así como el contexto económico en el que se encuentran para continuar sus estudios. 


\begin{tabular}{llcc}
\hline Tabla 1. Escuelas y cantidad de cuestionarios aplicados & Municipio & Cant. & Turno \\
\hline Escuela & Ascensión & 67 & Vespertino \\
\hline Colegio de Estudios Científicos y Tecnológicos 15 & Buenaventura & 46 & Matutino \\
\hline Preparatoria 8411 & Buenaventura & 58 & Matutino \\
\hline Colegio de Estudios Científicos y Tecnológicos 213 & Galeana & 37 & Matutino \\
\hline Colegio de Estudios Científicos y Tecnológicos 16 & Gómez Farías & 48 & Matutino \\
\hline Colegio de Estudios Científicos y Tecnológicos 10 & Gómez Farías & 17 & Matutino \\
\hline Centro de Bachillerato Tecnológico Agropecuario 214 & Janos & 11 & Vespertino \\
\hline Preparatoria & Nuevo Casas Grandes & 11 & Vespertino \\
\hline Preparatoria Bilingüe Lincoln & Ricardo Flores Magón & 55 & Matutino \\
\hline Colegio de Estudios Científicos y Tecnológicos 112 & Nuevo Casas Grandes & 298 & Matutino \\
\hline Centro de Estudios Tecnológicos, & Nuevo Casas Grandes & 123 & Matutino \\
Industrial y de Servicios 93 & Nuevo Casas Grandes & 23 & Matutino \\
\hline Instituto Blas Pascal & Nuevo Casas Grandes & 7 & Vespertino \\
\hline Preparatoria Francisco Villa & Total & $\mathbf{8 0 1}$ & \\
\hline Preparatoria Club de Leones & &
\end{tabular}

Fuente: Elaboración propia.

- Expectativas a futuro. Incluyeron preguntas que referían a las alternativas educativas que él o la estudiante tienen planeadas y la importancia de realizar estudios universitarios y su impacto en su vida futura.

- Iniciativa para el emprendedurismo. Esta parte se diseñó con la intención de comprender en qué medida se relacionan los estudios con la iniciativa de desarrollar sus propias ideas de negocios.

- Oferta educativa. Con esta variable se pretendió identificar el posicionamiento de las instituciones de educación superior de mayor interés y las intenciones de elección por algún programa de licenciatura o ingeniería.

Una vez recopilados los datos, se sistematizó con ayuda del software Statistics Program for Social Sciences (SPSS) versión 19. Se observó que los estudiantes sí mostraban interés por continuar los estudios en el nivel superior, lo cual motivó a la aplicación de un segundo cuestionario de solo cinco preguntas (febrero de 2015). El objetivo de esta nueva aplicación fue para identificar qué alternativas, áreas disciplinares y, más específicamente, qué licenciatura o ingeniería presentaban un grado tentativamente mayor de demanda por parte de los estudiantes. Ello permitió categorizar las diferencias existentes por género, edad y lugar de origen, principalmente. No obstante, para el presente texto solamente se presentan los resultados obtenidos de las variables relacionadas con los intereses educativos y las expectativas laborales y profesionales de los estudiantes en cuestión, indicando las tendencias entre hombre y mujeres.

\section{Rissurinos}

Antes de continuar con los resultados es necesario describir el perfil del estudiante 264 de bachillerato en la región. Los jóvenes son hijos de familia, pues viven con sus 
padres; no cuentan con un empleo y la actividad principal es ser estudiante. Provienen de familias relativamente pequeñas con no más de cuatro hermanos. Los gastos mensuales, promedio, ascienden a 500 pesos, los cuales son dedicados a la compra de materiales didácticos para sus clases. Son solteros y tienen en promedio 17 años, lo cual indica que no han reprobado ningún grado a lo largo de su trayectoria académica y que podrían ingresar al nivel superior apenas con la mayoría de edad, que es a los 18 años. A continuación, se expondrán los resultados obtenidos de acuerdo con las variables indicadas.

\section{Intereses edurotivivos}

Si bien una de las premisas centrales que motivó el presente estudio era conocer el posicionamiento de la Universidad Autónoma de Ciudad Juárez (UACJ), debido a que en los últimos años se ha incrementado de manera considerable la oferta de IES en la región. En el desarrollo de la misma fue posible detectar elementos de interés de los estudiantes y algunas perspectivas a futuro con una marcada diferencia entre hombres y mujeres, de ahí que se haya procurado definir mejor las variables a medirlas con base en el contexto local.

Por otro lado, puede considerarse que buena parte de las instituciones universitarias (públicas o privadas) ofrecen programas académicos similares, por lo que la oferta educativa es reducida entre sí. A ello se añade que aquellos que cuentan con recursos económicos más firmes optan por salir a estudiar a ciudades como Juárez o Chihuahua capital.

Con relación a quienes deciden continuar sus estudios en la región, según los resultados identifican en principio a la UACJ, la Universidad Tecnológica de Paquimé (UTP) y la Universidad Autónoma de Chihuahua (UACh) como las que prefieren. El Instituto Tecnológico de Nuevo Casas Grandes, así como la Escuela Normal Superior y la Pedagógica Nacional (UPN) son las menos reconocidas ${ }^{1}$ (véase figura 2).

El reconocimiento de la institución de educación superior de preferencia debe ir correlacionado con el programa de estudios al cual el estudiante es afín o que muestra un mayor interés por el mismo. En este caso, existen diferencias significativas entre las preferencias de las y los jóvenes de nivel medio superior, pues en el caso de las mujeres, la licenciatura de mayor preferencia es educación, seguida de enfermería $\mathrm{y}$ en un tercer lugar resalta la opción de ninguna.

Resulta por demás interesante que sean estas dos licenciaturas (educación y enfermería) a las que las mujeres se dirigen con mayor demanda. En primer lugar, puede ser debido a la construcción imaginaria de la protección y cuidados que debe estimular la mujer a los demás, así como el rol en el que se ha inculcado a las jóvenes desde niñas: enseñar y educar.

Además, desde el punto de vista económico, ambas profesiones cuentan con un modelo de inserción a la burocracia del estado que les permite prácticamente asegurar el futuro propio y de su familia, ya que contar con una plaza estatal o federal se puede realizar carrera profesional docente 0 en el sistema de salud, lo que permite el acceso a niveles de ingreso muy por encima de la media de la región, el cual, cabe decir, es apenas superior a los dos salarios mínimo al día. 


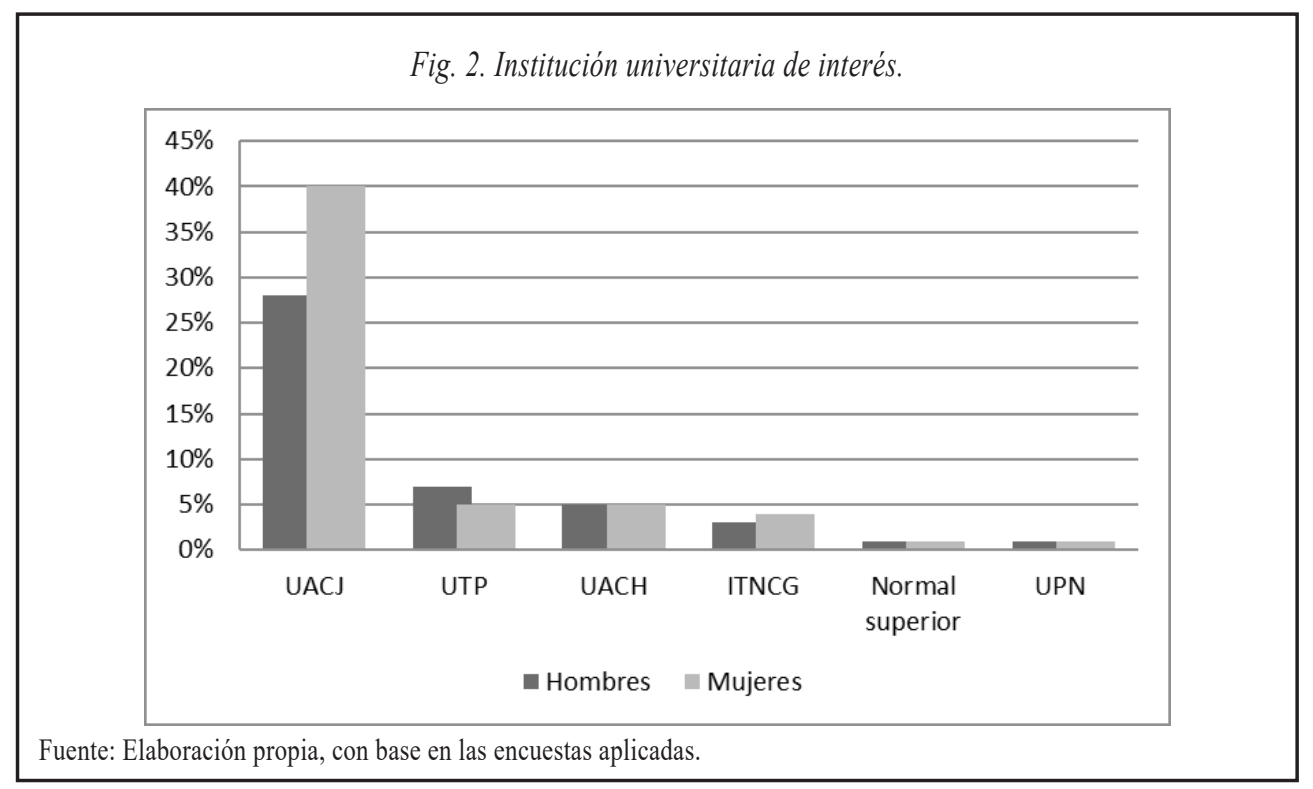

Lat cuestión de génerio

Al cuestionar a los estudiantes qué licenciaturas o ingenierías les gustarían que se ofertaran, las respuestas fueron variadas tanto en hombres como en mujeres, pues mientras unos eligieron relaciones exteriores (internacionales), otros optaron por educación especial. De nueva cuenta vuelve a manifestarse esa tendencia de las mujeres al área de la enseñanza y al cuidado de los otros. Los hombres mantienen la idea de profesiones relacionadas con la administración de empresas y la contabilidad como segunda opción; muy de cerca se encuentra la ingeniería en electrónica o sistemas de cómputo, así como la de ciencias agropecuarias y forestales.

En el caso de los hombres, resaltó que en el último semestre de su educación media superior aún no decidían qué opción educativa cursar en el nivel superior. En segundo lugar, se sitúa la licenciatura en educación; como tercera y cuarta opciones seleccionan disciplinas del área médica, como enfermería y médico veterinario (véase figura 3).

En el caso de las mujeres, después de educación especial se divide casi por parte iguales entre psicología clínica, odontología, gastronomía y turismo y derecho; en ningún caso aparece una mención a las áreas de ciencias exactas, donde pueden incluirse las ingenierías o las matemáticas ${ }^{2}$ (véase figura 4).

En suma, los intereses educativos de los estudiantes encuestados se orientan hacia las ciencias sociales y humanidades en el caso de las mujeres. Particularmente resaltan educación y enfermería, si bien una parte considerable de los encuestados omitió responder dicha pregunta. Lo anterior puede significar una falta de claridad de los estudiantes en relación a la licenciatura que deseen estudiar, por lo que es necesario reforzar los programas de orientación vocacional de las instituciones de bachillerato de la región para otorgarles mayores herramientas a sus futuros egresados 266 de los diversos programas académicos existentes en la región como fuera de esta. 

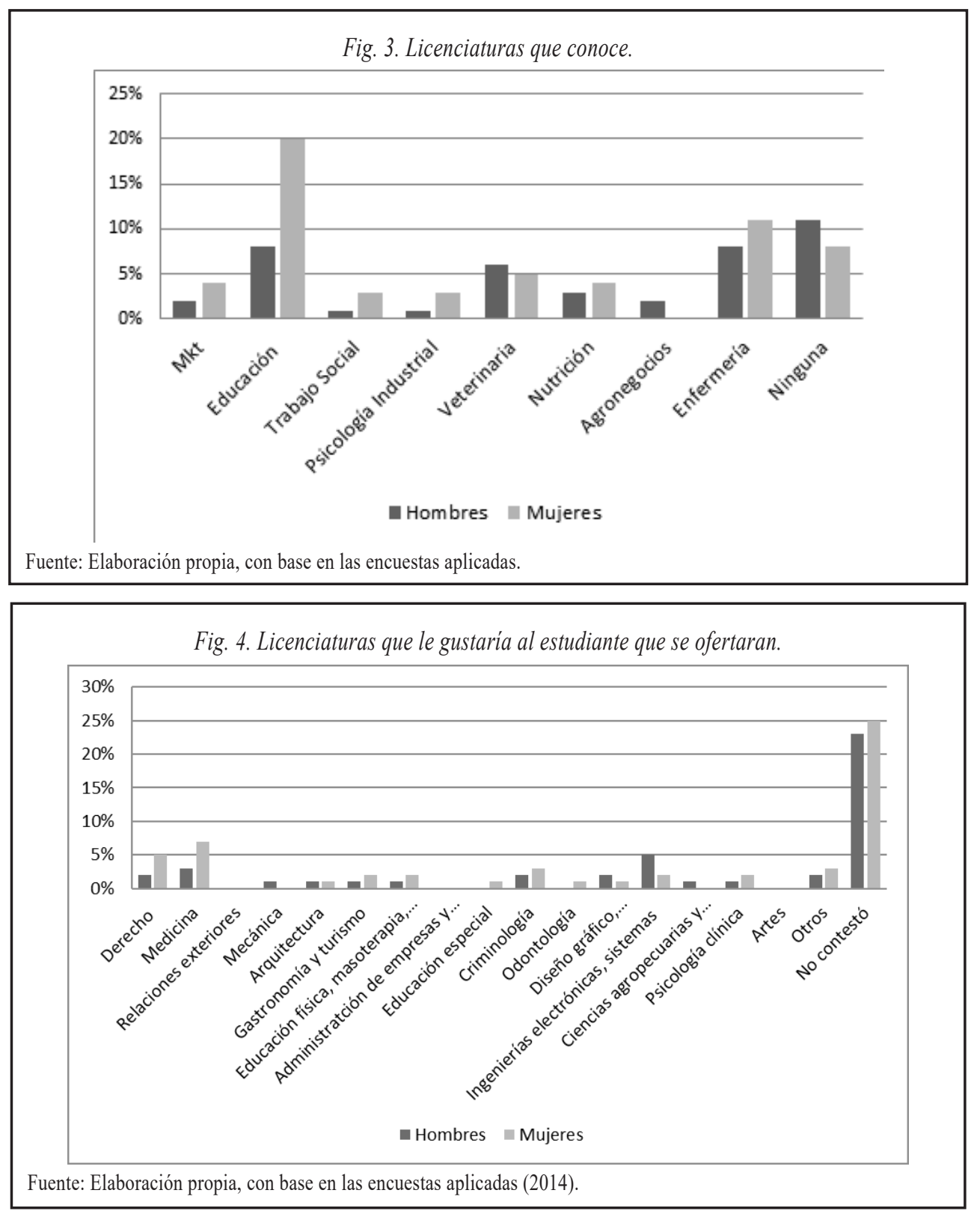

Por su parte, los hombres muestran menos interés en la licenciatura a estudiar, puesto que resalta su grado de indecisión. De tal manera que tampoco sorprende que a las mujeres les gustaría la oferta de licenciatura en educación especial y medicina, puesto que son programas educativos muy similares a los que ya conocen. En tanto que los hombres, prefieren relaciones internacionales, aunque también mencionan ingenierías; es decir, carreras opuestas a las señaladas por las mujeres.

\section{Expectativias laboriales y profesionales}

En un país donde el salario mínimo apenas es superior a los dos mil pesos mensuales y una región como la noroeste de Chihuahua que exhibe condiciones similares a la 
media nacional, culminar una carrera profesional puede representar una oportunidad importante para mejorar el ingreso personal y con ello aspirar a un nivel de vida mejor. Dicha premisa se intentó contrastar al preguntar a los estudiantes si contaban con un plan después de egresar del bachillerato. La mayor parte de los estudiantes respondieron de manera afirmativa la interrogante en cuestión; pocos son los que indicaron no contar con un plan al egresar del nivel medio superior. En ese sentido, el caso de los hombres son menos que las mujeres los que contestaron que no tienen planes (véase figura 5).

Lo anterior pone de manifiesto el desconocimiento de los estudiantes acerca del mercado laboral regional, así como las posibilidades de inserción dentro del mismo. De hecho, el sector terciario se configura como la mayor fuente de empleos, junto al sector primario, fundamentalmente en la producción de nuez, chile, durazno, sorgo y algodón. En menor grado existe una industria maquiladora fincada en Nuevo Casas Grandes, donde se elaboran arneses y asientos para camionetas de General Motors (véase figura 6).

Respecto a los resultados anteriores, cabe destacar que, a nivel regional, solamente existen tres museos, siendo el más importante el Museo de las Culturas del Norte, más conocido como Zona Arqueológica de Paquimé, ubicado en Casas Grandes, el cual es operado por el Instituto Nacional de Antropología e Historia (INAH). Adicionalmente, la región no cuenta con una gran prominencia de medios de comunicación, puesto que los diarios y las radiodifusoras son extensiones de medios fincados en Ciudad Juárez y Chihuahua.

Como ya se mencionó, los salarios a nivel nacional y en la región noroeste de Chihuahua son muy bajos, lo cual motivó la interrogante de qué remuneración sería la deseable obtener por parte de los estudiantes. La interrogante se introdujo en forma de rangos deseables de remuneración. En el nivel más bajo, el límite superior se fijó en tres mil 500 pesos; sucesivamente se incrementó el ingreso en alrededor de mil 500 pesos más por nivel hasta situarse en 10,000 o más.

Para las mujeres, el nivel salarial deseado oscila entre los tres mil 500 y los cinco mil pesos en su mayoría; es menor el número de aquellas que piensan obtener un salario superior a los 10 mil pesos. De manera inversa se encuentran los hombres,

Fig. 5. Plan de egreso del nivel medio superior.

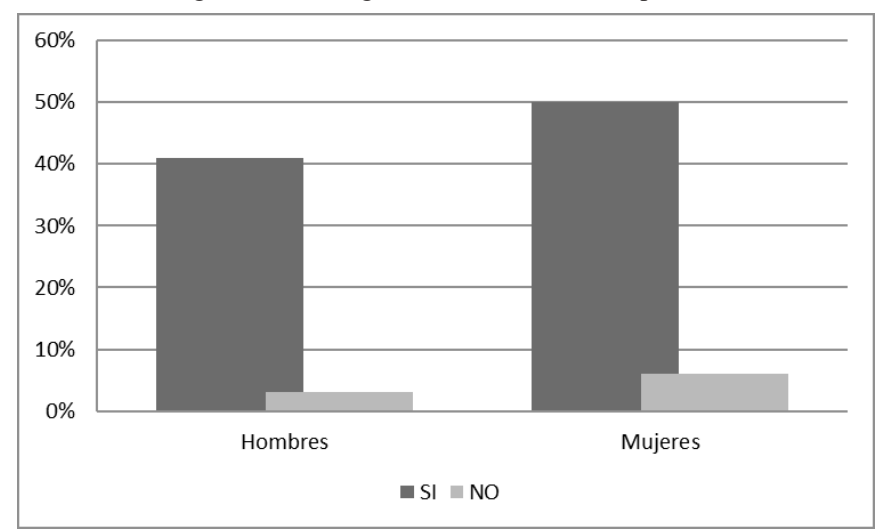

Fuente: Elaboración propia, con base en las encuestas aplicadas (2014). 


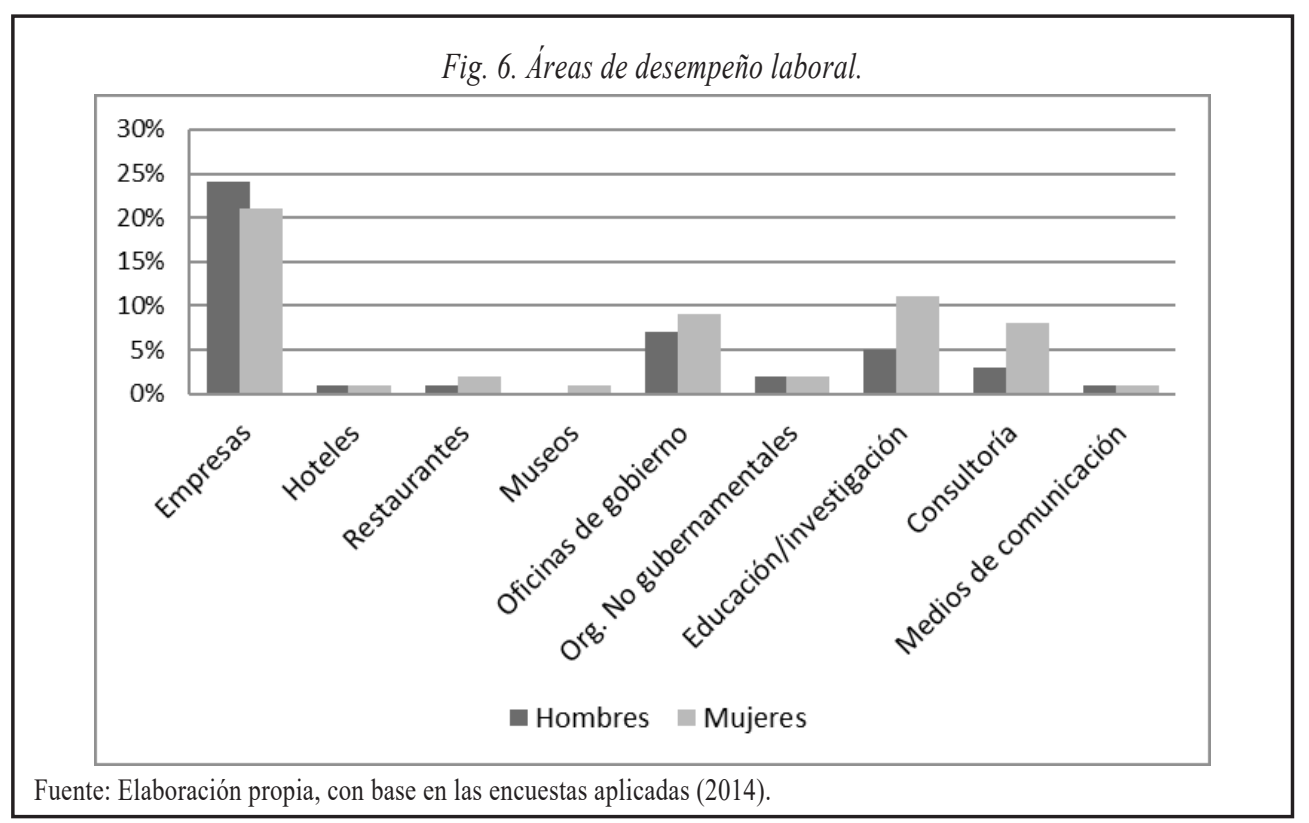

quienes definitivamente tienen como alternativa principal un ingreso superior a los 10 mil pesos y son los menos quienes piensan en rangos menores a los cinco mil pesos (véase figura 7).

Esta diferencia en cuanto al ingreso salarial entre mujeres y hombres puede deberse al escaso contacto con el mercado laboral que han tenido las mujeres a esa edad, pues al depender de una red fuerte familiar que la ayuda a solventar sus gastos, tienen poco conocimiento de las necesidades y gastos que se deben realizar dentro del entorno. Mientras que los hombres posiblemente tienen mayores responsabilidades fuera del hogar y en algunos casos pueden no contar con esa red de apoyo, lo que les hace entrar en contacto con el mercado laboral en edades más prematuras que las mujeres y, por tanto, exhiben una ambición mayor en términos de remuneración.

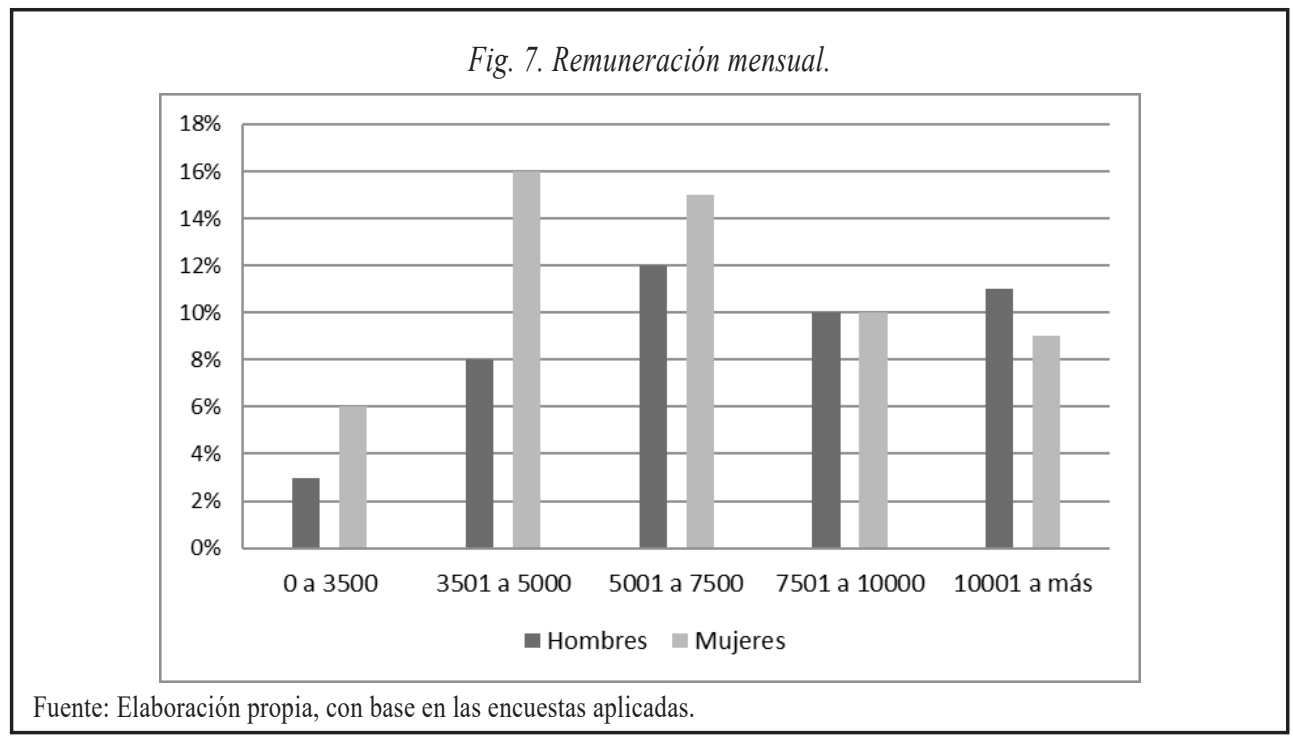


Otra cuestión determinante en este estudio era conocer el papel que los estudiantes otorgan a la licenciatura y en general a los estudios superiores como una herramienta catalizadora para acceder a un nivel de vida mayor. En esta cuestión, un poco menos del $40 \%$ de las mujeres, y un poco más del $60 \%$ de los hombres, consideraron que obtener la licenciatura no garantiza un mejor futuro. De manera afirmativa respondió alrededor del 55\% de las mujeres y un poco más del $40 \%$ los hombres (véase figura 8).

Ese mismo comportamiento se reafirma en el grado de importancia que otorgan los hombres a la obtención de un título universitario, ya que la mayoría de ellos lo consideran como nada importante, en tanto que las mujeres, de manera opuesta, lo consideran como muy importante, algo importante 0 importante la obtención del título o grado, lo que implica concluir los estudios universitarios (véase figura 9).

Esta puede ser una tendencia principal para explicar la baja demanda de estudios en instituciones de educación superior en la región, aunque también pueden existir de tipo sociocultural debido a que en ocasiones se considera de poca relevancia continuar los estudios universitarios, por lo que se por opta incorporarse al mercado de trabajo.

En cuanto a estudios de posgrado, las mujeres muestran una mayor inclinación por cursar un programa de maestría, aunque sus compañeros solo optarían por una especialidad a lo sumo, o quizá un diplomado; en último sitio se encuentra la maestría (ver figura 10). Este resultado, junto a los mostrados con anterioridad, exhiben una clara tendencia a que los hombres se interesan más por desarrollarse en ámbitos de vinculación con el sector productivo, por contar con ingresos más altos y sólidos, que continuar estudios o perspectivas que reflejen un desarrollo educativo.

En cambio, las mujeres tienen una inclinación mayor por el desarrollo académico, sin que ello signifique para ellas necesariamente la obtención de un ingreso mayor en el futuro, aspecto que suena contradictorio, puesto que, en el ideario, los estudios de posgrado deberían representar un escalón dirigido hacia la consolidación laboral y, por ende, la obtención de un ingreso mayor; sin embargo, dicha situación no parece reflejarse en las aspiraciones de las mujeres encuestadas.

Fig. 8. Mejor futuro con licenciatura.

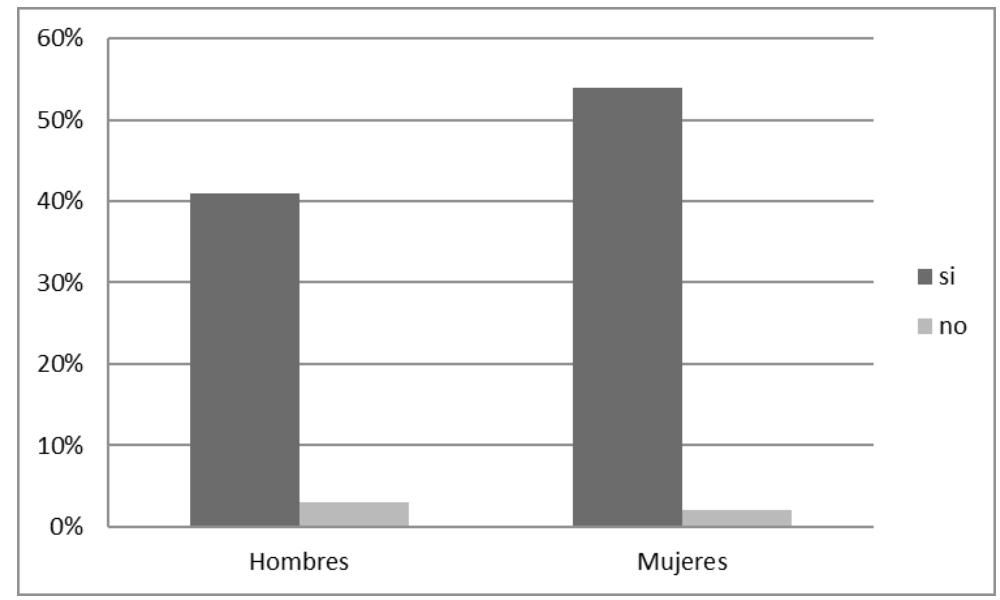

Fuente: Elaboración propia, con base en las encuestas aplicadas (2014). 

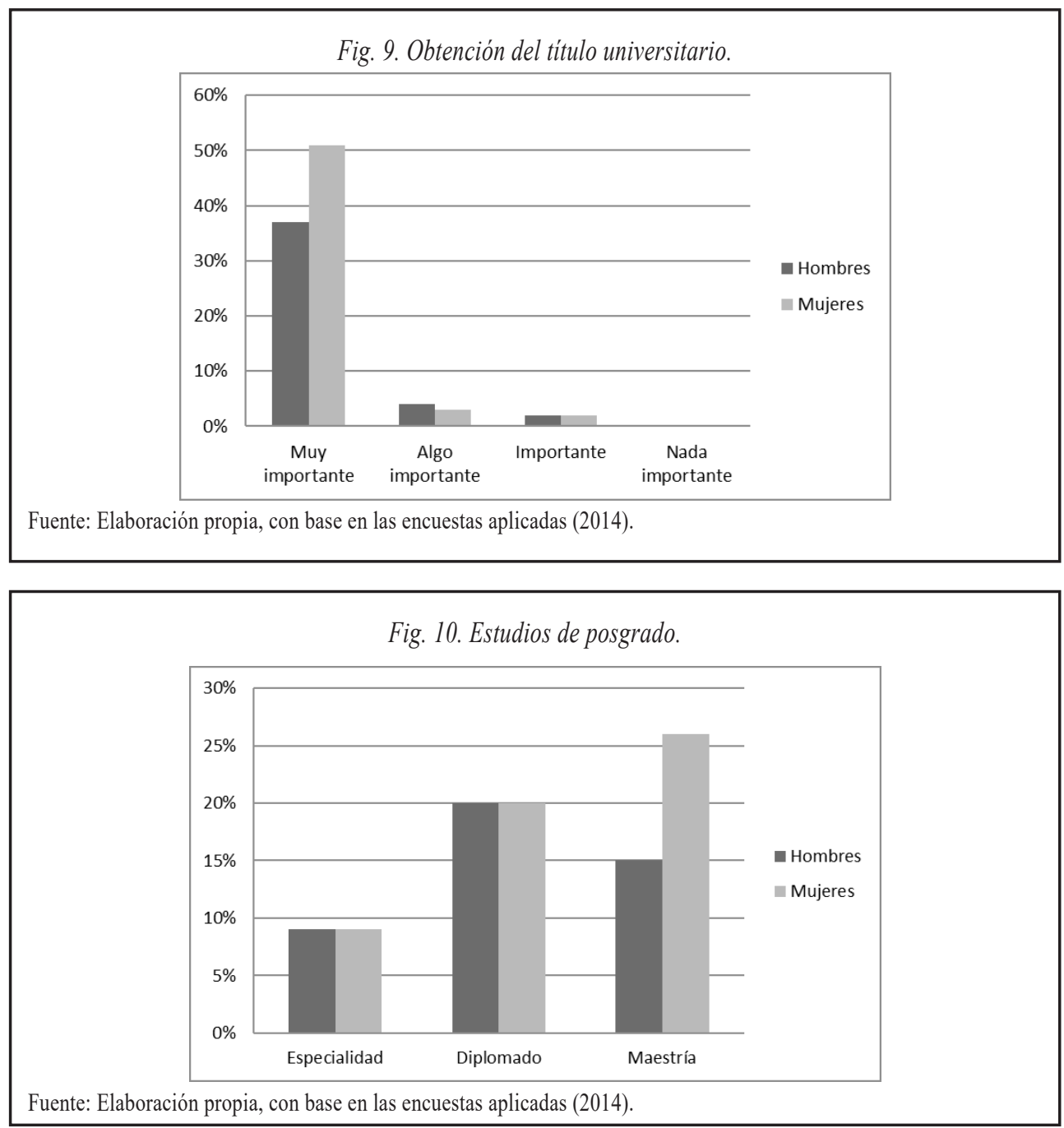

Conchisiones

Al ser la educación un bien público, y uno de los principales mecanismos de igualación de oportunidades a nivel social, garante del desarrollo de sociedades más justas e igualitarias, ha motivado que a nivel global y en México se amplíe la cobertura por parte del Estado hacia diversos niveles que anteriormente no se cubrían. En ese tenor, la inclusión de la educación media superior como obligatoria en nuestro país ha motivado un esfuerzo importante de la Secretaría de Educación federal y de las secretarías de Educación estatales en búsqueda de incrementar la cobertura de dicho nivel.

Lo anterior ha provocado un incremento notorio en el número de estudiantes inscritos en EMS y con ello también han surgido nuevos retos en la agenda de investigación. Uno de los retos identificados, y que se configura en la premisa básica de este estudio, corresponde a explorar con mayor detalle qué piensan los estudiantes en la antesala de ingresar al nivel profesional. Profundizamos sobre los intereses educativos, las expectativas laborales y profesionales en particular de los estudiantes 
de nivel medio superior de la región noroeste de Chihuahua, así como la influencia del género en ello.

Los principales hallazgos exhiben que los intereses educativos de las mujeres se orientan hacia programas vinculados a la educación y a la salud, lo cual en buena medida puede entenderse como un elemento propio de aseguramiento de su futuro y buena parte de mantener un estatus dentro de la región, pues se vincula a la incorporación del sector público, el cual brinda un número de prerrogativas laborales de mayor amplitud, a diferencia de profesiones como la contabilidad o los negocios, que son áreas de emprendedurismo.

A partir de los hallazgos de la presente investigación se siguiere una mayor orientación hacia los estudiantes desde su ingreso al nivel medio superior en relación a las condiciones laborales, así como las vocaciones productivas existentes en la región en la cual se desarrollan, en el sentido de dotar de toda la información disponible que les permitan analizar las implicaciones de la elección de un programa u otro en su desarrollo laboral y profesional en el futuro.

Queda pendiente dentro de la agenda de investigación efectuar un censo de todos los programas académicos que se ofertan en las diversas instituciones de educación superior que cuentan con presencia en la región para efectivamente analizar si los intereses educativos, laborales y profesionales de los estudiantes encuentran respaldo precisamente en dichas instituciones.

Lo anterior posibilitaría dos aspectos centrales que debe involucrar el desarrollo educativo de una región: las competencias para la inserción laboral y el desarrollo de negocios y la cooperación entre instituciones. En otras palabras, una mayor oferta de programas educativos sin tomar en cuenta las competencias que debe adquirir el futuro profesionista, así como el limitado impulso dentro de las instituciones hacia el emprendedurismo, solamente provoca una saturación de profesionistas que compiten por empleos escasos en una región económicamente deprimida, que presionan aún más hacia la baja en las remuneraciones.

Por tal razón, la cooperación entre las instituciones de educación superior debería convertirse en una condición sine qua non para que el sistema educativo de la región se articule hacia la conformación de un plan estratégico de formación de recursos humanos que posea competencias especializadas en el desarrollo de negocios basados en las fortalezas regionales que posibiliten el tránsito hacia un nivel de desarrollo mayor de los y las jóvenes de la región noroeste del estado de Chihuahua.

Dicho plan estratégico de cooperación interinstitucional es además necesario para también atender problemas específicos de los estudiantes al momento de incorporarse al nivel universitario, pues es común que una alta proporción de matriculados deserten en los primeros semestres. Lo anterior puede comprenderse mejor si se divide en tres momentos:

1. Apoyo en aspectos de orientación vocacional, donde la elección del programa educativo sea en realidad lo que el estudiante desea ser y hacer como parte de un proyecto de vida, pues en buena medida muchos de quienes ingresan no comprenden del todo cuál es el perfil de ingreso y menos aún el de egreso. 
2. Seguimiento a la trayectoria académica de los estudiantes, pues en ocasiones carecen de habilidades en áreas como las matemáticas, comprensión de lectura y hábitos de estudio, por mencionar algunas.

3. Apoyo a estudiantes a partir de un programa de tutoría donde puedan atender algunas de sus inquietudes, ya que, en ocasiones, además de su rol como universitario, deben atender otros problemas de tipo familiar que de manera indirecta afecta su desempeño escolar y en muchas ocasiones es uno de los factores que impacta en el ámbito de la permanencia, rezago y reprobación, que también afecta a las instituciones educativas. De ahí la importancia para que en futuras ocasiones se promueva el estudio de estos aspectos.

Es decir, para comprender mejor los intereses educativos y las expectativas laborales y profesionales de los estudiantes se debe considerar que en el último año del nivel medio superior los jóvenes deben tomar una decisión de suma importancia por continuar su formación académica, y en el primer año de la universidad se forja el sentido profesional y laboral, pues invertirá al menos cuatro años y medio de su vida a ello. Por lo que se debe comprender de manera más amplia todo aquello que rodea e impacta a los jóvenes.

\section{ReFprevirIIS}

Asociación Nacional de Universidades e Instituciones de Educación Superior (ANUIES).

(2013). Anuario de estadisticas educativas. Recuperado de http://www.anuies.mx/content. php?varSectionID $=166$

Banco Mundial. (2019). Gasto público en educación, total (\% en PIB). Instituto de Estadística de la Organización de las Naciones Unidas para la Educación, la Ciencia y la Cultura (UNESCO). Recuperado de https://datos.bancomundial.org/indicador/SE.XPD.TOTL.GD.ZS

Bautista-Flores, E., Sánchez-Carlos, O. y López Salazar, R. (2013). Encuesta tendencias educativas entre jóvenes bachilleres de la región noroeste de Chihuahua. Chihuahua: Universidad Autónoma de Ciudad Juárez, División Multidisciplinaria Nuevo Casas Grandes, Chihuahua.

Gobierno del Estado de Chihuahua. (2008). Análisis descriptivo de la estructura económica e industrial del estado de Chihuahua. Centro de Investigaciones Sociales y Económicos.

Gobierno del Estado de Chihuahua. (2009). Análisis descriptivo de la estructura económica e industrial del estado de Chihuahua. México. Recuperado de http://chihuahua.gob.mx/atach2/ codech/uploads/AN\%CILISIS\%20DESCRIPTIVO\%20DE\%20LA\%20ESTRUCTURA\%20 ECON\%D3MICA\%20E\%20INDUSTRIAL\%20DEL\%20ESTADO\%20DE\%20 CHIHUAHUA.pdf

Haq, M. (1999). Reflections on human development. Oxford University Press, Delhi.

Instituto Nacional para la Evaluación de la Educación (INEE). (2018). Panorama educativo de México. Indicadores del Sistema Educativo Nacional 2017. Educación básica y media superior. México. Recuperado de http://publicaciones.inee.edu.mx/buscadorPub/P1/B/116/ P1B116.pdf

Lewin, K. (1993). Education and development the issues and the evidence (education research paper n. 6). Centre for International Education University of Sussex.

López, R. y Bautista, E. (2015). Expectativas educativas y laborales de estudiantes de bachillerato en la región noroeste de Chihuahua. En Chihuahua hoy. Ciudad Juárez: Universidad Autónoma de Ciudad Juárez. Recuperado de http://elibros.uacj.mx/omp/index.php/ publicaciones/catalog/download/57/51/603-1?inline $=1$ 
Organización para la Cooperación y el Desarrollo Económicos (OCDE). (2015). Panorama de la educación 2015, México. Nota pais. Recuperado de https://www.oecd.org/mexico/Educationat-a-glance-2015-Mexico-in-Spanish.pdf

Secretaría de Educación Pública (SEP). (2012). Sistema educativo de los Estados Unidos Mexicanos. Principales cifras. Ciclo escolar 2011-2012. Recuperado de http://www.sep.gob. $\mathrm{mx} /$ work/models/sep1/Resource/1899/3/images/principales_cifras_2011_2012.pdf.

Serdyukov, P. (2017, abril 3). Innovation in education: what works, what doesn't, and what to do about it? Journal of Research in Innovative Teaching \& Learning, 10(1), 4-33. https://doi. org/10.1108/JRIT-10-2016-0007.

Sistema Nacional de Información Estadística Educativa. (2016). Escuelas, alumnos y maestros, según nivel educativo. México: Secretaría de Educación Pública. Recuperado de http://www. snie.sep.gob.mx/descargas/estadistica/SEN_estadistica_historica_nacional.pdf

UNESCO. (2007). Compendio mundial de la educación. Comparación de estadísticas de la educación en el mundo. Quebec, Canadá: Instituto de Estadística de la UNESCO. Recuperado de https://www.oei.es/historico/quipu/compendio educacion2007.pdf

UNESCO. (2017). Informe de seguimiento de la educación en el mundo, 2016: la educación al servicio de los pueblos y el planeta: creación de futuros sostenibles para todos. París. Recuperado de https://unesdoc.unesco.org/.../attach_import_784bebdd-f932-4a42-bfe2$7 \mathrm{ab} 6 \mathrm{ccf} 12311$

Villarreal, E. (2018). Endogeneidad de los rendimientos educativos en México. Perfiles Latinoamericanos, 26(51), 265-299. https://dx.doi.org/10.18504/p12651-011-2018

\begin{abstract}
Notis
${ }^{1}$ Los resultados de la institución educativa de preferencia pueden estar sesgados, debido que, al comunicar las instrucciones para el llenado de las encuestas a los estudiantes, se mencionaba como parte de un proyecto más amplio de la División Multidisciplinaria de la UACJ en Nuevo Casas Grandes orientado a medir el posicionamiento de la misma a nivel regional.

${ }^{2}$ El reactivo contenía las opciones descritas en el gráfico, por lo que un alumno podía escoger todas las opciones, una o dos, etcétera. Ello implica que la cuantificación permite identificar cuáles programas académicos son los más atractivos para el estudiante, a pesar de mostrar una preferencia mayor hacia un programa determinado.
\end{abstract}

